\title{
Space-qualified laser system for the BepiColombo Laser Altimeter
}

\author{
Reinald Kallenbach, ${ }^{1, *}$ Eamonn Murphy, ${ }^{2}$ Bodo Gramkow, ${ }^{3}$ Markus Rech, ${ }^{4}$ \\ Kai Weidlich, ${ }^{4}$ Thomas Leikert, ${ }^{4}$ Reiner Henkelmann, ${ }^{4}$ Boris Trefzger, ${ }^{4}$ Bodo Metz, ${ }^{4}$ \\ Harald Michaelis, ${ }^{5}$ Kay Lingenauber, ${ }^{5}$ Simone DelTogno, ${ }^{5}$ Thomas Behnke, ${ }^{5}$ \\ Nicolas Thomas, ${ }^{6}$ Daniele Piazza, ${ }^{6}$ and Karsten Seiferlin ${ }^{6}$ \\ ${ }^{1}$ Max Planck Institute for Solar System Research, Max-Planck-Strasse 2, 37191 Katlenburg-Lindau, Germany \\ ${ }^{2}$ European Space Agency, Directorate of Technical and Quality Management TEC-MME, Optoelectronics, \\ ESA, ESTEC, Keplerlaan 1, 2201 AZ Noordwijk ZH, The Netherlands \\ ${ }^{3}$ European Space Agency, Directorate of Science and Robotic Exploration, ESA, ESTEC, \\ Keplerlaan 1, 2201 AZ Noordwijk ZH, The Netherlands \\ ${ }^{4}$ Cassidian Optronics GmbH, Carl-Zeiss-Strasse 22, 73447 Oberkochen, Germany \\ ${ }^{5}$ German Aerospace Center, Institute of Planetary Research, Planetary Sensor Systems, \\ Rutherfordstrasse 2, 12489 Berlin, Germany \\ ${ }^{6}$ Physikalisches Institut, University of Bern, Sidlerstrasse 5, 3012 Bern, Switzerland \\ *Corresponding author: kallenbach@mps.mpg.de \\ Received 12 April 2013; revised 12 October 2013; accepted 4 November 2013; \\ posted 19 November 2013 (Doc. ID 188725); published 16 December 2013
}

\begin{abstract}
The space-qualified design of a miniaturized laser for pulsed operation at a wavelength of $1064 \mathrm{~nm}$ and at repetition rates up to $10 \mathrm{~Hz}$ is presented. This laser consists of a pair of diode-laser pumped, actively $q$-switched Nd:YAG rod oscillators hermetically sealed and encapsulated in an environment of dry synthetic air. The system delivers at least 300 million laser pulses with $50 \mathrm{~mJ}$ energy and $5 \mathrm{~ns}$ pulse width (FWHM). It will be launched in 2017 aboard European Space Agency's Mercury Planetary Orbiter as part of the BepiColombo Laser Altimeter, which, after a 6-years cruise, will start recording topographic data from orbital altitudes between 400 and $1500 \mathrm{~km}$ above Mercury's surface. () 2013 Optical Society of America

OCIS codes: (140.0140) Lasers and laser optics; (140.2010) Diode laser arrays; (140.3330) Laser damage; (140.3550) Lasers, Raman.

http://dx.doi.org/10.1364/AO.52.008732
\end{abstract}

\section{Introduction}

Laser range finding is a well-established technology applied in a number of ground-based instruments [1-4]. The requirements for a space-borne laser altimeter are driven by firm constraints on available power and mass resources, environmental conditions on board the spacecraft, by the aim for very high reliability, and in particular by tight link budgets for ranging

$1559-128 \mathrm{X} / 13 / 368732-15 \$ 15.00 / 0$

(C) 2013 Optical Society of America over large distances. Experience on laser altimetry from space has already been established in the course of the past four decades. The lunar surface had been explored with space-borne altimeter systems from the Apollo 15, 16, and 17 spacecraft [5], as well as the Clementine [6], the SELENE [7], and, very recently, from the Lunar Reconnaissance Orbiter [8], the Chandrayaan-1 [9], and the Chang'E-1 [10] missions. The terrestrial surface has been investigated from space by the Shuttle Laser Altimeter [11] and the Geoscience Laser Altimeter [12]. The Martian surface has been very successfully mapped with the Mars 
Observer Laser Altimeter [13], the asteroid Eros has been explored with the NEAR Laser Ranging Investigation [14], and first data are available from Mercury's surface [15] from the MESSENGER Laser Altimeter (MLA) $[16,17]$.

European Space Agency's (ESA's) BepiColombo mission to Mercury, to be launched in 2017, will complement NASA's MESSENGER mission (MErcury Surface, Space ENvironment, GEochemistry and Ranging) already launched in 2004 and inserted into orbit around Mercury in 2011. Many of the science goals of the two missions, such as the detailed investigation of Mercury's surface and magnetic field, and their interaction with the Hermian exosphere and the solar wind are similar [18-20]. However, the orbits of the two missions are quite different. MESSENGER, including MLA, is in a highly elliptical orbit with a periapsis argument of $\approx 60^{\circ}$ and altitudes ranging from 200 to $15,000 \mathrm{~km}$ and, therefore, mainly mapping the Northern hemisphere by imaging and altimetry, as NASA's Mariner 10 already mapped the Southern hemisphere of Mercury in 1974/75 [21]. BepiColombo includes two spacecraft, the Mercury Magnetospheric Orbiter (MMO) by JAXA and the Mercury Planetary Orbiter (MPO) by ESA. MMO's orbit has peri- and apoherm distances of $400-16,000 \mathrm{~km}$, and its scientific payload concentrates on magnetospheric and solar wind observations. MPO, carrying the BepiColombo Laser Altimeter (BELA), orbits at lower altitudes ranging from $400 \mathrm{~km}$ to only $1500 \mathrm{~km}$. On its near-polar orbit, with an argument of periapsis initially at the equator, it allows for a dense global coverage of Mercury's surface with laser altimeter ground tracks. While offering advantages for the coverage of Mercury's surface with altimetry records, the fairly low MPO orbit implies a strong exposure of the spacecraft and its scientific payload to the heat radiation of Mercury.

BELA will become the first laser altimeter on a planetary mission of ESA [22-25]. It will form an integral part of a larger geodesy and geophysics package on board the MPO, incorporating a radio science experiment [26], an accelerometer [27], a magnetometer [28], and a stereo-imaging camera [29]. The combination of gravity field, magnetic field, and static and time-dependent topography data provides insight into the geology and interior structure of the planet and in turn into the formation and evolution history of Mercury, its interaction with the interplanetary magnetic field and into the dynamo process in Mercury's partially liquid core [30]. A challenging goal in this context is the determination of the size of the liquid core from measurement of the tidal amplitude of Mercury's solid surface due to solar gravity with less than $10 \%$ uncertainty [31]. This tidal signal is estimated to be of order one meter [32] and is correlated with the solar radiation onto Mercury and the MPO spacecraft during the 88-day eccentric orbit of Mercury around the Sun. Consequently, systematic errors in the measurement data caused by thermoelastic bending or deformation of the mechanical structures carrying the optical components of the BELA instrument must be excluded. This poses one of the many demanding requirements on the BELA hardware, both on the laser system as well as the receiver optics. Typically, the pointing distortion due to thermoelastic bending of an optical component of the BELA laser must not exceed a few $\mu$ rad within the operating temperature range, and the distance changes between critical lenses must not exceed a few micrometers. Larger distortions would cause variations in the optical performance parameters of the BELA laser such as beam profile, divergence and pointing, as well as pulse energy and temporal pulse shape. This in turn would have an impact on the quality of the distance measurement [33] or could even lead to a complete failure of the instrument due to misalignment of the transmitter against the receiver.

In this article, we describe the very stable thermomechanical design of the BELA laser system, the diodelaser pumping scheme that can operate efficiently over a wide temperature range, as well as the approach of encapsulating all laser optics into a dry synthetic air environment, thus avoiding the expected reduction of the damage threshold for contamination initiated laserinduced damage under vacuum operation relative to air [34]. The qualification test results [35] are updated at the end of the manufacturing phase of the engineering and qualification model (EQM) after implementation of last design optimizations.

\section{Design}

\section{A. Requirements from the Link Budget}

The BELA instrument (Fig. 1) is a bistatic system.

This means, the transmitter and receiver are separate systems, which has the practical advantage for parallel development at different locations $[22,36,37]$. The main design driver for both the transmitter and the receiver is the challenging link budget to be met by the BELA instrument over the large distance between the MPO spacecraft and Mercury's surface. The number $N_{r}$ of photons returned to the avalanche photodiode detector is given by [38]

$$
N_{r}=\left(\frac{E_{t} \eta}{h \nu}\right) \cdot\left(\frac{A_{r}}{d_{M}^{2}}\right) \cdot t_{\mathrm{sys}} \cdot t_{\mathrm{atm}}^{2} \cdot\left(\frac{r_{\mathrm{tar}}}{\Omega_{\mathrm{tar}}}\right) .
$$

The parameters of this equation are given in Table 1 .

The most significant requirement for the laser is the pulse energy $E_{t} \approx 50 \mathrm{~mJ}$ in order to ensure a sufficient number of returned photons on the detector, which has an area of $A_{r} \approx 0.049 \mathrm{~m}^{2}$. The number of background detectable photons due to the thermal radiation of Mercury is reduced by using an optical filter in the detection chain having a bandwidth of less than $1 \mathrm{~nm}$ and by reducing the field of view (FOV) of the detector telescope to an angular width of about $400 \mu \mathrm{rad}$. Consequently the number of detected photons can be as low as $N_{r} \approx 20$ to guarantee a low probability of false detection [42]. Using the parameters of Table 1 , this should enable the 

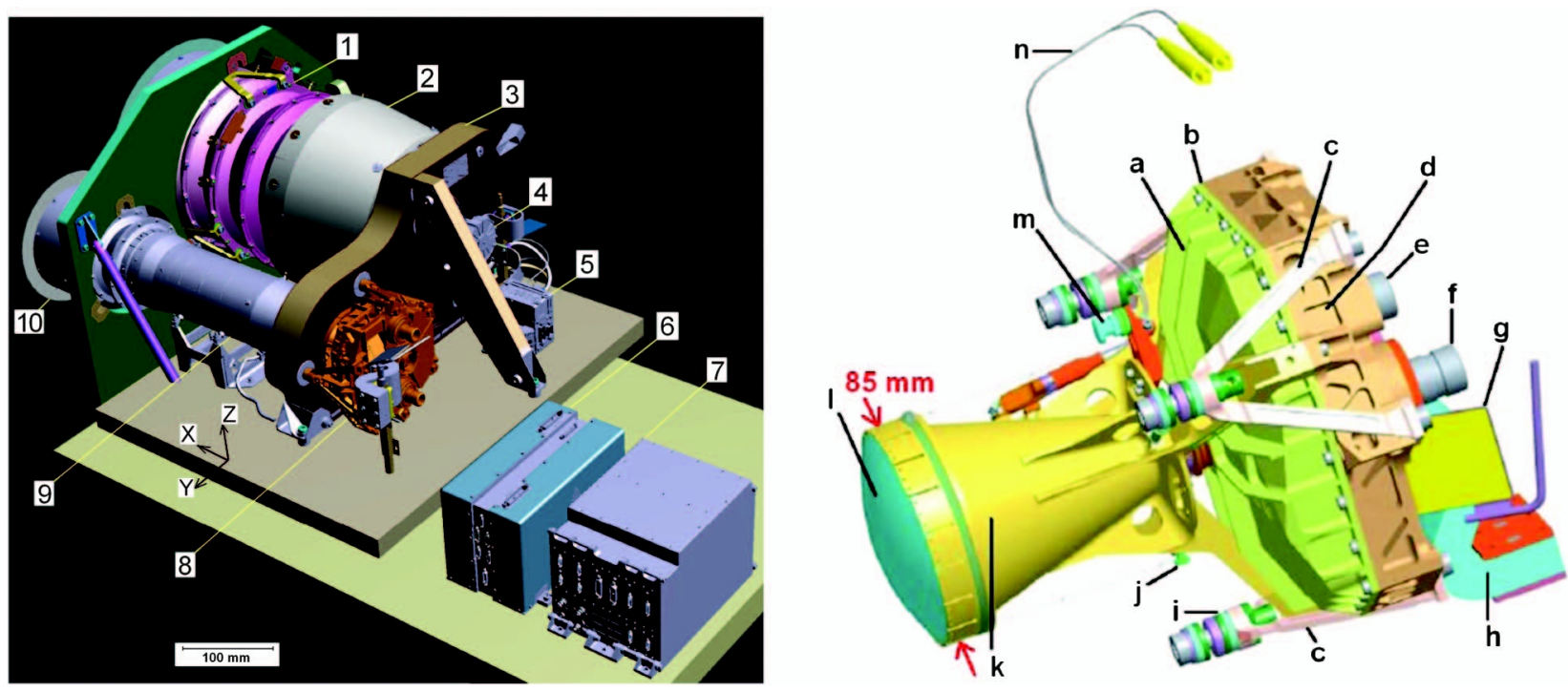

Fig. 1. Left: BELA laser transmitter integrated with the receiver [22]. 1, Receiver baffle unit [23]; 2, receiver telescope covered by multilayer insulation; 3, carbon-fiber-enforced aluminum honeycomb baseplate; 4, focal plane assembly; 5 , analog electronics unit of the receiver; 6 , laser electronics unit (LEU); 7, electronics unit including power converter module, data processing module, and rangefinder module; 8, laser head box (LHB); 9, stray light protection unit; 10, transmitter baffle unit. Right: CAD drawing of the BELA LHB with a, pressurized (1 bar) laser compartment cover made of aluminum beryllium metal alloy (AlBeMet162); b, C-ring sealing interface; c, quasiisostatic mounts (bipods); d, laser compartment base made of AlBeMet162; e, data line connector; f, power line connector for $200 \mu \mathrm{s}$ diode laser current pulses at $100 \mathrm{~A} ; \mathrm{g}$, heater bracket at LHB thermal interface; h, thermal strap to spacecraft cold finger; i, stack of spherical washers and shims for quasi-isostatic precision mounting onto the BELA instrument baseplate; $\mathrm{k}$, beam expander (BEX) cone; 1 , apsherical BEX front lens; m, optical reference plane; n, fiber-optical interface for laser start pulse to avalanche photodiode detector of the receiver.

extraction of reliable altimetry data at MPO altitudes of at least up to $1055 \mathrm{~km}$ [42]. The link margin increases with the inverse square of spacecraft altitude, which allows for measurements in areas where Mercury has a surface reflectivity lower than $25 \%$ when the MPO spacecraft is at lower altitudes than $1055 \mathrm{~km}$. At $400 \mathrm{~km}$ altitude, the margin of the link budget is more than a factor of 6 .

An obvious and essential requirement is that the laser spot on Mercury's surface falls within the detector's FOV. Several effects, such as the variation of

Table 1. Link Budget Parameters of the BELA Instrument

\begin{tabular}{|c|c|}
\hline Parameter & Value \\
\hline$E_{t}$, laser pulse energy $[\mathrm{mJ}]$ & $>50$ \\
\hline$\tau_{P}$, laser pulse length [ns] & $3-8$ \\
\hline Lifetime [Mshots] & $>300$ \\
\hline$\Delta \Phi$, full-cone beam divergence [ $\mu \mathrm{rad}]$ & $50 \pm 10$ \\
\hline$\lambda=c / \nu$, wavelength $[\mathrm{nm}]$ & $1064 \pm 0.3$ \\
\hline$d_{M}$, distance to Mercury $[\mathrm{km}]$ & $400-1500$ \\
\hline$\Omega_{\mathrm{tar}}$, surface scattering angle & $\pi$ \\
\hline$A_{r}$, detector area $\left[\mathrm{m}^{2}\right]$ & 0.049 \\
\hline$t_{\text {sys }}$, instrument transmission & 0.77 \\
\hline$\delta E_{t}$, energy stability $[\mathrm{mJ}]$ & $<2.5^{a}$ \\
\hline$f_{P}$, pulse repetition rate $[\mathrm{Hz}]$ & $1-10$ \\
\hline$E_{t}$ at end of life $[\mathrm{mJ}]$ & $>40$ \\
\hline$\delta \Phi$, bore-sight stability [ $\mu \mathrm{rad}]$ & $<50$ \\
\hline$h \nu$, photon energy $\left[10^{-19} \mathrm{~J}\right]$ & 1,875 \\
\hline$t_{\mathrm{atm}}$, transmission of atmosphere & 1 \\
\hline$r_{\mathrm{tar}}$, diffuse reflectivity & 0.25 [39-41] \\
\hline$\Delta \Psi$, field of view $[\mu \mathrm{rad}]$ & $4 \overline{00}$ \\
\hline$\eta$, detector quantum yield & 0.36 \\
\hline
\end{tabular}

${ }^{a} 1 \sigma$ over 1000 pulses spacecraft speed and its distance relative to Mercury's surface in addition to the thermoelastic distortion of the baseplate and the detector telescope, need to be considered in the establishment of the pointing stability budget. Ultimately, the bore-sight of the laser itself needs to be stable over the whole mission time within $50 \mu \mathrm{rad}$ with respect to the baseplate to which it is mounted. Extracting the most relevant parameters from the wealth of details on laser altimetry $[33,43]$, we also conclude that the laser beam divergence should not be larger than the bore-sight stability range. On the other hand, a beam divergence below a critical minimum value leads to undesirable speckle effects. This constrains the beam divergence to between 40 and $60 \mu \mathrm{rad}$, corresponding to a laser beam diameter on Mercury's surface of between 16 and $90 \mathrm{~m}$.

\section{B. Technical Requirements}

The technical requirements basically apply for all instruments on board the MPO spacecraft, where the available mass and power resources must be shared in the most efficient way possible. Resources and environmental conditions for the BELA laser system mounted onto the BELA instrument baseplate on board MPO are summarized in Table 2 . Additionally, stringent requirements on electromagnetic interference and magnetic cleanliness apply. The latter requirement is given by the fact that precise measurements of the interplanetary magnetic field and Mercury's planetary magnetic field are to be taken from the MPO platform [28]. Typically, the static magnetic moments of the instrument units of BELA 
Table 2. Resources of the BELA Laser System Aboard the MPO and Environmental Parameters at the Laser Head Box (LHB)

\begin{tabular}{lc}
\hline Parameter & Value \\
\hline Mass of LHB [kg] & 1.883 \\
Mass of laser electronics unit $[\mathrm{kg}]$ & 2.837 \\
Electrical input power [W] & $<22.5$ \\
Cruise and separation shock loads $[\mathrm{g}]$ & 1000 \\
LHB operational temperature range $\left[{ }^{\circ} \mathrm{C}\right]$ & $5-45$ \\
LHB structural design loads, $3 \sigma[\mathrm{g}]$ & 200 \\
\hline
\end{tabular}

have to be less than $20 \mathrm{mAm}^{2}$, and the total $\mathrm{AC}$ magnetic moments at frequencies above $30 \mathrm{~Hz}$ need to be lower than $1 \mathrm{mAm}^{2}$.

\section{Technical Realization}

The development of the dedicated BELA laser system design inherited major aspects of reliable range finders at Carl Zeiss Optronics, now Cassidian Optronics in Oberkochen, Germany [44]. The actual hardware consists of two boxes, the lightweight laser head box (LHB; Fig. 2, left) isostatically mounted onto the BELA instrument baseplate (Fig. 1) and the laser electronics unit (LEU; Fig. 2, right) mounted onto the spacecraft bench, providing laser voltage and current supply as well as control communication for the LHB on one side and a commanding interface to the electronics unit of the BELA instrument on the other side.

\section{Optical and Electrical Design}

The LHB contains a primary and a redundant, single-stage, actively $q$-switched, diode laser sidepumped Nd:YAG rod laser system with unstable resonator geometry (Fig. 3, left). Each of the two Nd:YAG laser rods inside the LHB is pumped by four laser diode assemblies (LDAs) R-8 manufactured by
SemiConductor Devices (SCD). They emit at about $808 \mathrm{~nm}$ wavelength with an electrical-to-optical efficiency of about $50 \%$. The four LDAs are driven in series by 100 A diode laser current pulses with a length of approximately $200 \mu$ s supplied from a $300 \mathrm{~V}$ capacitor reservoir (Fig. 2, right). Their pump light is directed to the laser rod for absorption of its major part during first pass transmission. The remaining part of the pump light is contained by diffusing reflectors made from purified polytetrafluorethylen material. This cavity (Fig. 4, right) is optimized to homogeneously distribute the $808 \mathrm{~nm}$ pump light over the laser rod volume achieving maximum pump light energy density inside the rod.

A $3 \mathrm{kV}$ voltage across a Pockels' cell is switched to actively open the optical path in the laser resonator for stimulation of laser radiation at $1064 \mathrm{~nm}$. During its few round trips, the stimulated laser beam pulse at $1064 \mathrm{~nm}$ expands in diameter. This allows for subsequent stimulation of radiation from the entire laser rod volume. A variable reflectivity outcoupling mirror by INO, Canada, determines the mode profile and establishes a near Gaussian mode shape of the laser pulse leaving the resonator. The optical-tooptical efficiency for the conversion of the pump light into the laser radiation is about $10 \%$. In order to extract the stored energy at this high efficiency, the $q$-switch needs to transit from nonpolarizing to quarter-wavelength condition within at most $30 \mathrm{~ns}$. The corresponding electronic element had to be miniaturized to fit into the LHB in close proximity to the laser resonator. No space-qualified MOSFET switch is available in this high-voltage range. Therefore, eight transistors IRHNJ67C30 have been cascaded, and their gates are driven synchronously at different voltage levels by carefully trimmed highfrequency transformers. With this active $q$-switch,
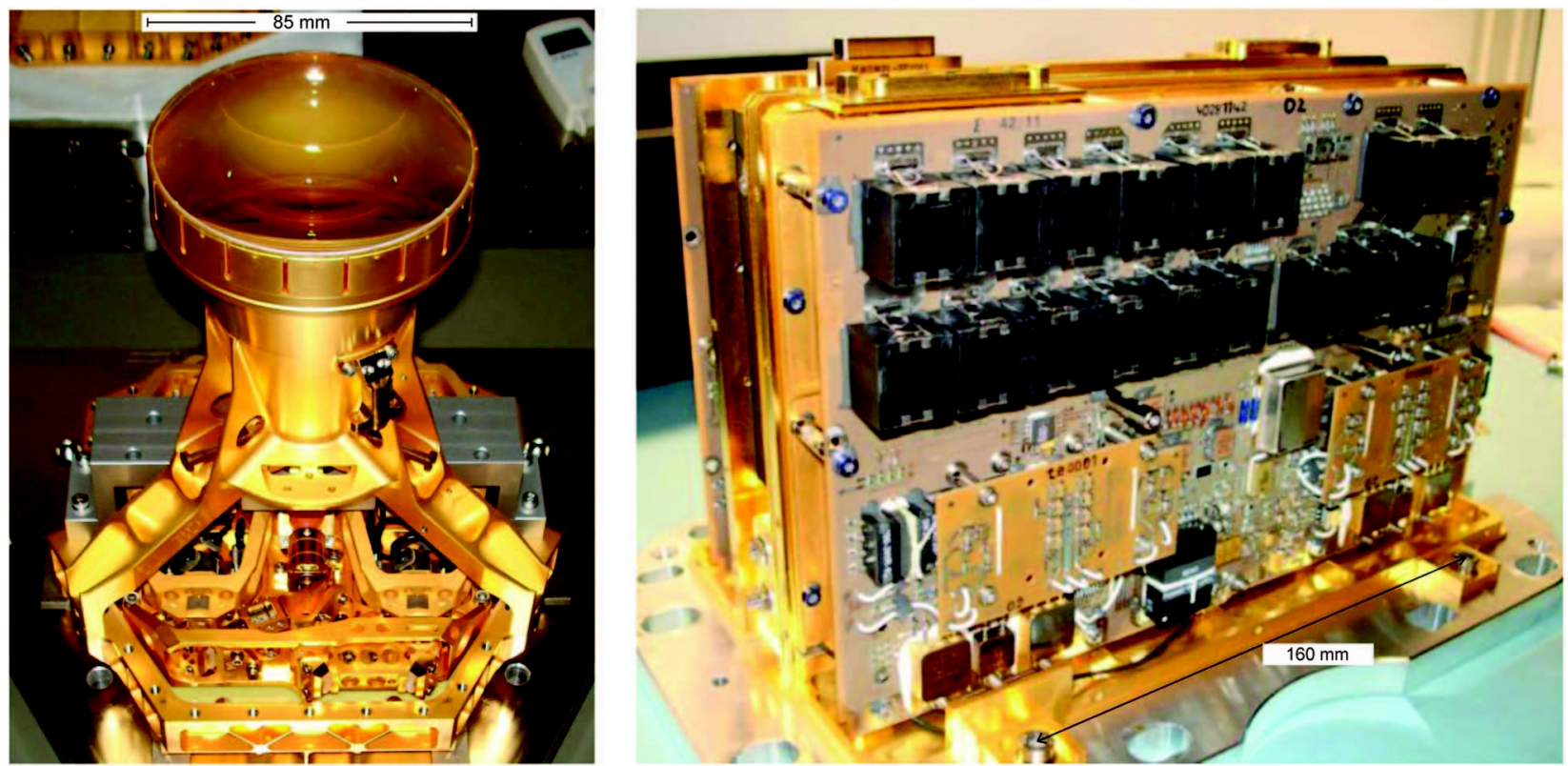

Fig. 2. Left: EQM of the BELA LHB without cover and C-ring sealings containing two cold-redundant laser systems and including BEX optics. Right: EQM LEU with capacitor reservoirs to drive the 100 A diode laser current at pulse lengths of up to $200 \mu \mathrm{s}$. 

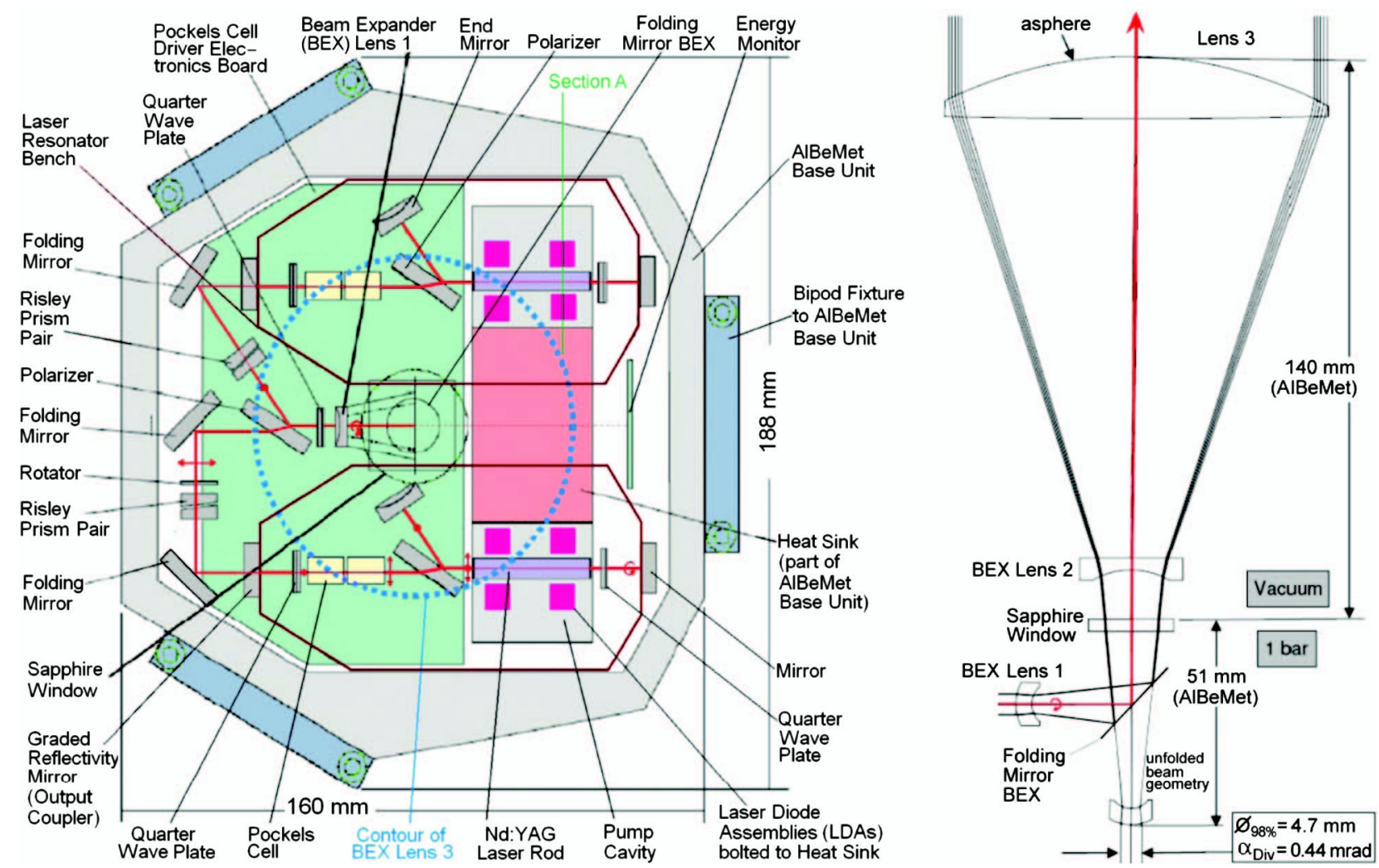

Fig. 3. Left: schematics of the two redundant, orthogonally polarized BELA laser resonators and the transfer optics to the BEX. The cross-section A is detailed in Fig. 4 (right). Right: schematics of the BEX optics with its first lens inside, and the second and third lens outside the pressurized volume, respectively.

typical pulse durations of $5 \mathrm{~ns}$ are achieved at $1064 \mathrm{~nm}$, which is appropriate for the altimetry application.

To protect laser optics from laser-induced damage effects, the LHB is pressurized with synthetic air and

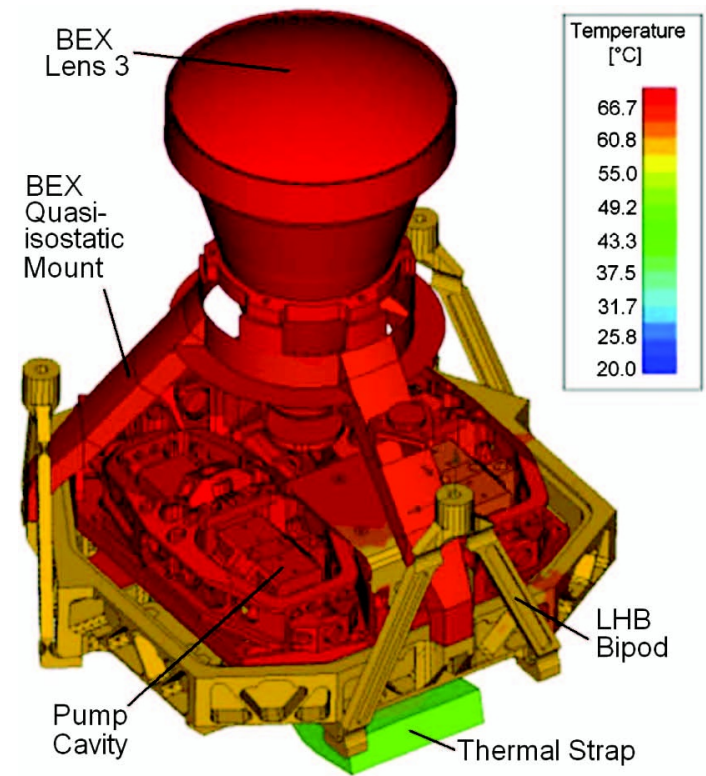

sealed by C-rings facilitating a pressure drop of no more than 100 mbars over the 12 -year mission time. Additionally, a catalyst convertor inside the LHB housing reduces concentration of contamination near the laser optics. The decision to encapsulate

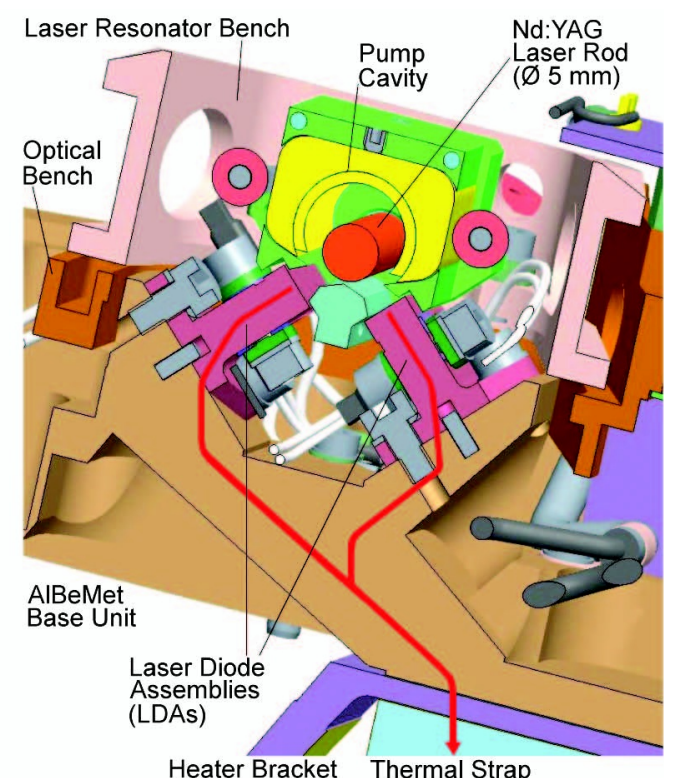

Fig. 4. Left: LHB with removed cover and color-coded temperature distribution as derived through an ESATAN simulation software package. The calculated temperature distribution was applied to the structural mathematical model for thermoelastic analysis. Right: CAD cross section, corresponding to cross-section A of Fig. $\underline{3}$ (left), through one of the BELA laser pump cavities indicating the heat flow from the LDAs to the thermal strap. 
the laser into dry synthetic air has been taken right at the beginning of the project, for several reasons: (1) spectroscopy experiments with the frequencydoubled [45] radiation of a highly frequencystabilized, continuous-wave dye laser at $486 \mathrm{~nm}$ [46] already revealed the problems with damage of dielectric coatings of an optical built-up cavity in vacuum [47] in the 1980s. (2) Detailed experimental studies on laser-induced contamination and damage on dielectric coatings in vacuum have been mostly performed for space laser applications at UV wavelengths of $355 \mathrm{~nm}[34,48,49]$; however, the studies suggest that similar processes may cause, although to a lesser extent, damage on laser optics in vacuum when exposed to high intensities of $1064 \mathrm{~nm}$ radiation. (3) Encapsulating all laser optics into a dry synthetic air environment has already been applied in the frame of the CALIPSO Aerosol Lidar Mission [50]. The CALIOP laser began initial flight operation under a chamber air pressure of 1.24 bar. The BELA laser is the first encapsulated laser to be launched on a planetary mission.

The beam expander (BEX) optics (Fig. 3, right) comprise three lenses and a sapphire window between the first and second lens through which the laser beam exits the pressurized LHB volume. The first BEX lens next to the laser resonator serves as a compensator to the residual aberrations and initial misalignment of the BEX elements. The 85-mm diameter aspherical BEX front lens is made from radiation hard Schott LF5G15 glass. It should be noted that the BEX is complemented by a sapphire window in the laser baffle (Fig. 1, left) providing thermal shielding. The outgoing laser beam has a divergence of 40-60 $\mu \mathrm{rad}$ after leaving the BEX and reaches Mercury's surface with spot sizes between 16 and $90 \mathrm{~m}$. An optical fiber leading to the BELA receiver is connected to the BEX, providing a reference to the outgoing laser pulse and the start signal for the altimetry measurement.

\section{Structural and Thermal Design}

Launch vibrations, spacecraft separation shocks, and rapid change of thermal conditions during the $2.33 \mathrm{~h}$ orbit around Mercury are critical for laser alignment stability. Therefore, thermal interfaces have been separated from alignment-critical mechanical interfaces in order to reduce thermomechanical deformations of the latter. The LHB (Fig. 1, right) is mounted onto the BELA instrument baseplate by quasiisostatic mounts in form of bipod flexures (Fig. 4, left). These bipods have a low thermal conductivity of $0.02 \mathrm{~W} / \mathrm{K}$ and transfer only very small torques to the instrument baseplate, less than $0.04 \mathrm{Nm}$ over the full operational temperature range, in order to avoid deformations. The bipods are V-shaped and very flexible perpendicular to the plane of the "V" due to thinnings near their mounting interfaces. The mounting interface to the instrument baseplate contains a set of spherical washers allowing the release of residual stress during bolting. Ideally, each of the three bipod mounting interfaces only transfers forces perpendicular to the instrument baseplate.

The LHB structure has been machined from a lightweight, very stiff aluminium beryllium metal (AlBeMet162) alloy, with a density of $\approx 2.1 \mathrm{~g} \mathrm{~cm}^{-3}$ and elastic modulus of $\approx 180 \mathrm{GPa}\left(1 \mathrm{~Pa}=1 \mathrm{Nm}^{-2}\right)$ in order to keep the LHB mass within limits acceptable for the BELA instrument design. The thermal expansion coefficient of AlBeMet $\left(\approx 13.9 \mathrm{ppm} \mathrm{K}^{-1}\right)$ is lower than that of aluminum and its thermal conductance is very high $\left(\approx 210 \mathrm{~W} \mathrm{~m}^{1} \mathrm{~K}^{-1}\right)$. This further reduces critical torques and forces caused by thermal expansion of the LHB, which could be transferred to the BELA instrument baseplate via the bipods. Vibration eigenfrequencies of design elements inside the LHB have been kept at much higher frequencies than those typically arising from instrument and spacecraft excitations. The only eigenfrequency of the stand-alone LHB system below $2 \mathrm{kHz}$ is determined by the bipods as spring elements and the LHB main compartment as lumped mass. This intrinsic fundamental eigenmode at about $275 \mathrm{~Hz}$ has a quality factor of about 40; however, it couples to modes of the carbon-fiber-enforced aluminum honeycomb baseplate of BELA, resulting in somewhat damped coupled modes with quality factors of less than 20.

The pointing of the BELA laser with respect to the receiver is mainly determined by the pointing of the BEX optics (Fig. 1, right). This resulted in a precision design with the BEX supported by quasi-isostatic mounts (Fig. 4, left) attached to the base unit of the LHB near its bipod fixation to the instrument baseplate. This solution separates critical optics from the deformations of the box, which are, in turn, induced by thermal expansion and pressure differences between the intern and the extern of the laser box. The BEX is additionally wrapped in multilayer insulation foil in order to minimize radiative heat transfer. The BEX optics have no redundancy because of their size and mass, and have received special attention as elements of single point failure.

The stringent thermo-optical stability requirements on laser source and transfer optics to the BEX are fulfilled dividing the LHB in three thermally decoupled areas. The laser resonator is mounted on a dedicated bench (Fig. 5), which is thermally decoupled from the rest of the unit, in particular from the heat-dissipating laser diodes. The laser resonator is mounted on the optical bench, which represents the second thermal area. The optical bench hosts the transmitter optics and is made of the highly thermally conductive AlBeMet alloy in order to minimize thermal gradients and consequent deformations. The last thermal area consists of the base unit made of AlBeMet as well, as mentioned above. Heat dissipating elements, such as laser diodes (Fig. 4, right), laser rod, and electronics, are mounted on and efficiently coupled with this structural element. This base unit is thermally decoupled from the optical bench. A flexible link with high thermal conductance connects 


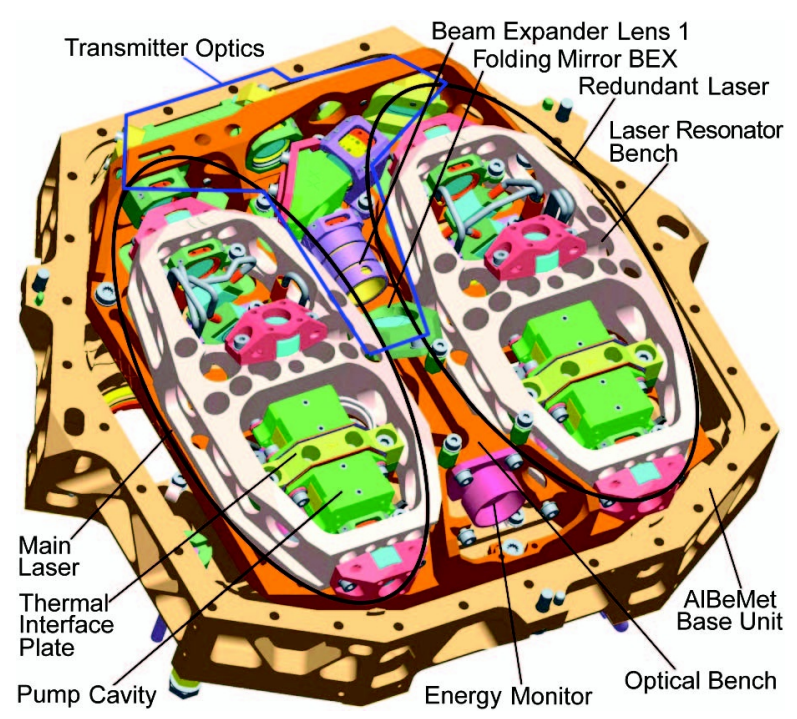

Fig. 5. BELA laser CAD drawing of the LHB with cover removed.

the external surface of the base unit with the heat sink (Fig. 4, left).

An additional heat source of the LHB/BEX system is the $1.5 \mathrm{~W}$ thermal radiation absorbed from the thermal environment outside the spacecraft and transferred from the transmitter baffle unit onto the BEX front lens, which is located some distance from the thermal link. Therefore, the thermomechanical behavior of the LHB including the BEX has been analyzed in detail. Based on the coefficient of thermal expansion (CTE) data for the BEX tube and the lens materials and by including the axial temperature distribution indicated in Fig. 4, a $2 \mu \mathrm{rad}$ increase of beam divergence was calculated. If the temperature distribution inside the BEX front lens material and the temperature dependence of its refractive index are taken into account this effect is actually compensated. The laser pointing error caused by temperature effects does not exceed $10 \mu \mathrm{rad}$ because the laser resonator and the transmission optics reside on a structure completely made from the same AlBeMet material as described above.

\section{Verification}

\section{A. Space Qualification of the Pump Diode Lasers}

High reliability is a key requirement for the use of diode lasers for space applications. The wear-out lifetime $\tau$ of the LDAs R-8 by SCD has been experimentally established from dedicated accelerated life tests at various increased operation temperatures and at several elevated electrical drive currents [51]. The tests at different temperatures lead to the so-called activation energy $E_{a}$ in eV. This parameter is a measure to describe the scaling of the lifetime, $\tau(T)$, at different operation temperatures according to the relation [드]

$$
\tau(T)=\tau\left(T_{0}\right) \exp \left[\frac{E_{a}}{k_{B}}\left(\frac{1}{T}-\frac{1}{T_{0}}\right)\right],
$$

where $k_{B}=8.63 \times 10^{-5} \mathrm{eV} \mathrm{K}^{-1}$ is Boltzmann's constant, and $T_{0}$ is some reference temperature at the diode laser junction. The LDA lifetime, $\tau$, is assumed to describe an exponential power degradation as a function of time $t$ at fixed temperature $T$ :

$$
P(t, T)=P_{0}(T) \exp \left[-\frac{t}{\tau(T)}\right] .
$$

Operation time $t$ can be replaced by the number of laser shots if the diode laser current and pulse duration are kept fixed. No interface temperatures to the diode laser heat sinks higher than $80^{\circ} \mathrm{C}$ could be realized. Therefore, the five test groups were not only operated at $70^{\circ} \mathrm{C}$ and at $80^{\circ} \mathrm{C}$ with $100 \mathrm{~Hz}$ repetition rate but also at $80^{\circ} \mathrm{C}$ applying increased repetition rates of 175,200 , and $250 \mathrm{~Hz}$. This way, the junction temperature can be further increased despite the limited heat sink base temperature of $80^{\circ} \mathrm{C}$. The scaling of junction temperatures with pulse repetition rate has been calibrated by measuring the wavelength shift of the diode lasers of typically $0.22 \mathrm{~nm}$ per ${ }^{\circ} \mathrm{K}$.

Sixty MShots at a repetition rate of $100 \mathrm{~Hz}$ have been applied in order to accumulate a large number of shots comparable to the mission requirement of 300 MShots, to keep the duration of the test campaign within reasonable limits, and to be able to observe measurable degradations. Pulsed operation at a repetition rate of $100 \mathrm{~Hz}$ has been assumed to be representative for pulsing at $10 \mathrm{~Hz}$ because the most important thermal time scales for heat transport from the diode laser junctions to the heat sink are of order 2-6 ms. This estimate is based on the thermal diffusivities of GaAs, $\kappa_{\mathrm{GaAs}}=2.6 \times 10^{-5} \mathrm{~m}^{2} \mathrm{~s}^{-1}$, or the heat spreader material $\mathrm{BeO}, \kappa_{\mathrm{BeO}}=6.7 \times 10^{-}$ $5 \mathrm{~m}^{2} \mathrm{~s}^{-1}$, combined with typical dimensions $d \approx 4 \times$ $10^{-4} \mathrm{~m}$ to $\tau_{\text {thermal }} \approx d^{2} \kappa^{-1} \approx 2 \ldots 6 \mathrm{~ms}$.

Some of the results of these so-called temperature step stress tests are summarized in Table $\underline{3}$ and in Fig. 6 , where it should be noted that the degradation data have much larger uncertainties and statistical variation than the junction temperature data. At junction temperatures of $432.4 \mathrm{~K}$, one of the four diode laser stacks failed completely so that it is not clear whether Eqs. (2) and (3) still apply at such high temperatures, i.e., whether there is a new type of failure mechanism. At the four lower temperatures applied in the test, degradations are rather small so that it can be assumed that $t \ll \tau(T)$, and temper-

\begin{tabular}{|c|c|}
\hline Temperature $\left[{ }^{\circ} \mathrm{K}\right]$ at Junction & Degradation after 60 Mshots [\%] \\
\hline 374.8 & $-0.16 \pm 0.15$ \\
\hline 384.8 & $+0.36 \pm 0.9$ \\
\hline 408.6 & $-1.57 \pm 1.0$ \\
\hline 416.5 & $-0.68 \pm 0.20$ \\
\hline 432.4 & $-35 \pm 38$ \\
\hline
\end{tabular}
ature variations are small compared to the absolute 


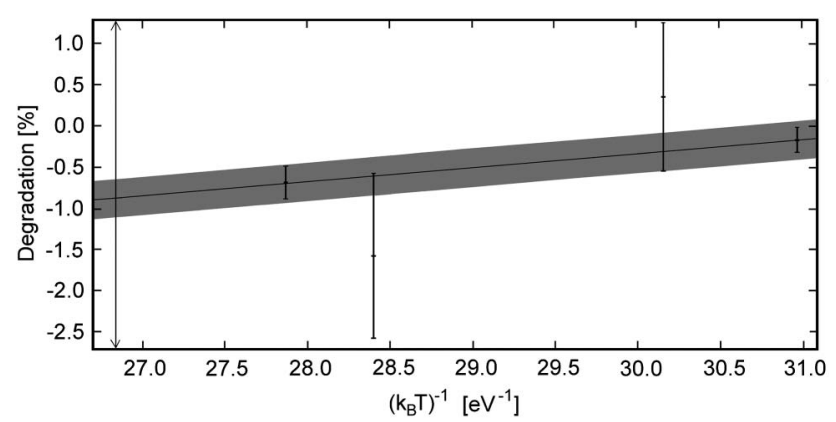

Fig. 6. Determination of the activation energy of the BELA diode laser assemblies from step stress analysis data. A $\chi^{2}$-fit with $63 \%$ confidence limit yields a slope of $(0.00169 \pm 0.00005) \mathrm{eV}$ and an abscissa intercept of $(-0.054 \pm 0.0015)$. The center of the ordinate, corresponding to a reference temperature $T_{0}$ to apply the linearization of Eq. (4), can be reliably determined as $29.3 \pm 1.3$. This leads to a degradation at $T_{0} \approx 400 \mathrm{~K}$ of $0.0045 \pm 0.0022$ as best fit. This value introduces the dominant relative uncertainty of $\approx 50 \%$ in the determination of the life time and the activation energy $E_{a}=(0.46 \pm 0.23) \mathrm{eV}$.

temperature values, $\left|T-T_{0}\right| \ll T_{0}$. Equations and ( $\underline{3})$ can then be linearized to

$$
\frac{P(t, T)-P_{0}(T)}{P_{0}(T)} \approx-\frac{t}{\tau\left(T_{0}\right)}\left(1-\frac{E_{a}}{k_{B} T}+\frac{E_{a}}{k_{B} T_{0}}\right)
$$

and it is straightforward to derive the two parameters $\tau\left(T_{0}\right)$ and $E_{a}$ including their uncertainties (Fig. 6). The lifetime $\tau(400 \mathrm{~K})$, equivalent to about $(13 \pm \overline{6})$ GShots, is compatible with the BELA application. During the BepiColombo mission, junction temperatures much lower than $400 \mathrm{~K}$ are to be expected so that the diode laser lifetimes may even be longer. Typically $330 \mathrm{~K}$ have to be expected. During short "hot" phases, the junction temperatures may rise to $\approx 340 \mathrm{~K}\left(67^{\circ} \mathrm{C}\right)$. The activation energy $E_{a}=$ $0.46 \pm 0.23 \mathrm{eV}$ is in the normal range for III-V single quantum-well diode lasers and has been studied in more detail elsewhere [51]. Further tests at increased diode laser currents up to $150 \mathrm{~A}$ have been performed to demonstrate safe operation at increased diode laser drive currents of $100 \mathrm{~A}$ needed for the BELA application [51].

However, as can be seen from the data in Fig. 6, the lifetime $\tau(T)$ and the activation energy $E_{a}$ do not describe the degradation behavior that well. These parameters only describe the typical degradation of the specific III-V semiconductors but no degradations that may be related, for instance, to the stack design. An additional degradation process commonly called "infant mortality" has been observed during the extended life test (ELT) at component level of eight LDAs of the so-called evaluation group up to 500 MShots. This ELT has been carried out at only $10 \mathrm{~Hz}$ pulse repetition frequency (PRF), i.e., fully representative for the real situation during the BepiColombo mission. Considering the rather hot environment of Mercury, some margin in operational temperature has been required. Therefore, the heat sink temperature of the LDAs during their ELT has been set to a rather high value of $75^{\circ} \mathrm{C}$. At $10 \mathrm{~Hz} \mathrm{PRF}$, the junction temperature is $2-4 \mathrm{~K}$ above the heat sink temperature, yielding a junction temperature of about $80^{\circ} \mathrm{C}$.

It has not entirely been resolved whether the rather high junction temperature in combination with the low PRF is responsible for the strong degradation of the LDAs during the first 20 MShots (Table 4). In some cases, many emitters of one or two of the eight bars in one LDA fail for reasons not entirely understood. Any hypothesis, such as degradation by subcritical crack growth [53] due to thermal or vibration stresses, or stresses introduced by handling could not be confirmed experimentally with statistical significance. The "infant mortality" has not been found clearly correlated with any of the other qualification tests performed with these LDAs, such as vibration, thermal cycling, or shock [54]. Therefore, the risk for this degradation had to be considered as unavoidable and needed to be mitigated.

The BELA laser life model, containing flightrepresentative optics and LDAs, has just passed the required 300 Mshots of operation at $10 \mathrm{~Hz}$ repetition rate. This test has been performed with a diode laser current of $100 \mathrm{~A}$ applied over $125 \mu \mathrm{s}$ for each pulse and heat sink temperatures of $56^{\circ} \mathrm{C}$ (Fig. 7), corresponding to junction temperatures of approximately $60^{\circ} \mathrm{C}$. These life test data show a phenomenon compatible with that of "infant mortality" of diode laser bars. When present, this typically occurs during the first 10-20 MShots of LDA operation and could result in a degradation of order $10 \%$ of one of the two redundant BELA laser systems. To avoid the occurrence of such "infant mortality" in any of the BELA laser flight hardware modelsalthough it could be compensated by increasing the pump time for the Nd:YAG laser rod-a burn-in over 20 MShots is performed during the assembly of the flight hardware. These burn-in procedures for both the flight model (FM) and the flight spare (FS) model have since been performed. The "infant mortality" is limited to at most $5 \%$ for each of the individual 16 FM and FS LDAs.

In addition to the tests briefly described above, the LDAs from SCD have undergone an extensive screening and evaluation test program including neutron and gamma irradiation, vibration, mechanical shock, voltage breakdown test, destructive

Table 4. Average Pulse Energy Degradation Data from the Extended Lifetime Test at Component Level with Eight BELA LDAs R-8 by SCD Belonging to the Evaluation Group

\begin{tabular}{lccc}
$\begin{array}{l}\text { Number of } \\
\text { Shots }\left[10^{6}\right]\end{array}$ & $\begin{array}{c}\text { Relative Pulse } \\
\text { Energy }\end{array}$ & $\begin{array}{c}\text { Number of } \\
\text { Shots }\left[10^{6}\right]\end{array}$ & $\begin{array}{c}\text { Relative Pulse } \\
\text { Energy }\end{array}$ \\
\hline & 1 & 100 & $0.864 \pm 0.139$ \\
20 & $0.884 \pm 0.117$ & 200 & $0.833 \pm 0.137$ \\
40 & $0.884 \pm 0.117$ & 300 & $0.824 \pm 0.131$ \\
60 & $0.883 \pm 0.122$ & 400 & $0.824 \pm 0.131$ \\
80 & $0.868 \pm 0.112$ & 500 & $0.820 \pm 0.132$ \\
\hline
\end{tabular}




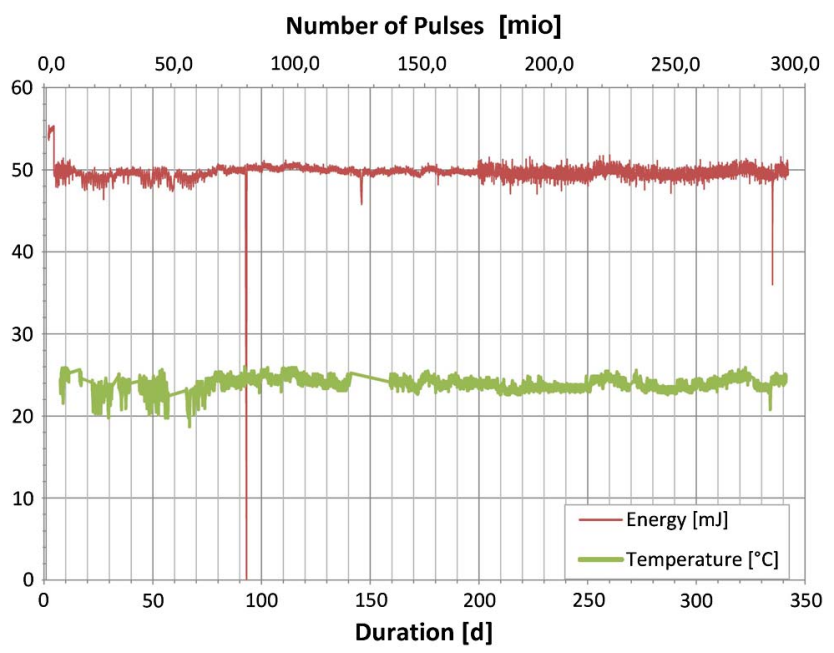

Fig. 7. BELA laser life test results. Three power drop-outs are visible: the first (at about 92 days) has been caused by a power blackout, the second (at about 142 days) by a drop-out of the laboratory temperature control, the third (at about 335 days) by drop-out of the synthetic air supply on 2 April 2013. The initial power degradation after about four Mshots is presumed to be caused by "infant mortality." (Note, that for practical reasons, this life model does not have the final C-ring sealing and, therefore, has a higher leakage rate than the EQM and the flight hardware. Although this leakage rate is negligible for contamination exchange, the life model has been kept in a vacuum chamber flushed by additional dry synthetic air during this life test).

physical analysis, storage test, and electro-optical performance testing.

We would like to draw attention to our specific approach for proton irradiation testing. Commonly, testing for the effects of damage due to particle irradiation in space environments is performed with accelerator facilities providing some fixed beam energy. This, however, neglects the fact that both damage and penetration depth of energetic particles in solids are strongly dependent on particle energy $[55,56]$. For instance, energy loss by electronic stopping of protons or other nuclei in solids is strongest at energy-per-mass ratios of roughly $100 \mathrm{keV} / \mathrm{amu}$. Nuclear stopping, however, peaks at energy-per-mass ratios of a few $\mathrm{keV} / \mathrm{amu}$. The penetration depth of $6 \mathrm{MeV}$ protons is of the order of a few hundreds of micrometers, while the penetration depth of $100 \mathrm{keV}$ protons is of the order of $500 \mathrm{~nm}$. The spectra of space particle radiation usually cover both the energy-permass ranges for maximum electronic stopping power and maximum nuclear stopping. A proton beam with $6 \mathrm{MeV}$ energy per particle may well pass a thin optics layer without causing major damage by nuclear stopping.

Consequently, we have taken a conservative approach by performing several irradiations at different proton energies and fluences. The LDAs had been irradiated by 70,130 , and $190 \mathrm{keV}$ protons in order to cause maximum potential damage by nuclear stopping inside the facet on the surface of the LDAs. A $6 \mathrm{MeV}$ proton beam has been applied in order to reach the single quantum well layer, which is at a depth of a few hundreds of micrometers, where the material needs to withstand the highest current and optical radiation densities, but not to overshoot these areas.

Table $\underline{5}$ gives an overview of the degradation in diode laser power after irradiation by proton beams with different energies and fluences. The tests have been based on the reference fluence of $1.15 \times 10^{11} \mathrm{~cm}^{-2}$ protons at $10 \mathrm{MeV}$ energy corresponding to $60 \mathrm{kRad}$ (Si). This is the expected radiation dose onboard the MPO spacecraft in course of the BepiColombo mission behind $2 \mathrm{~mm}$ of $\mathrm{Al}$ shielding. At their location inside the BELA LHB, the LDAs will only be exposed to about $10-20 \mathrm{kRad}$ depending on mission duration. This means, that the LDAs have been qualified for higher radiation doses than required for the BepiColombo mission; however, these results are encouraging for applications in space missions with more hostile radiation environment than Mercury's.

\section{B. Space Qualification of Laser Optics}

All resonator components have successfully passed the tests of military standard MIL-C-48497, as well as irradiation tests up to doses of $200 \mathrm{kRad}$ of both gammas and protons each, and laser-induced damage threshold (LIDT) tests [57] according to the international standard EN ISO 11254-2 (Fig. 8). (Note that a new LIDT test standard 21254-2:2011 has been introduced after finalization of the LIDT tests for the BELA laser components.) Spectral transmission tests at component level were supplemented with laser level tests for the laser rod and the $q$-switch before and after irradiation. It is beyond the scope of this paper to report on all details emanating from these tests. However, we would like to emphasize some of the most critical issues.

At the beginning of the BELA project, concerns have been raised about the radiation hardness of the rubidium titanyl phosphate (RTP) crystal implemented as active $q$-switch. It has proven to be fairly radiation hard, and may even be suitable for application up to a total dose of several hundred kRad (Fig. 9, left). The BELA laser energy degraded by $2.6 \%$ after $200 \mathrm{kRad}$ of gamma irradiation and in total by $3.4 \%$ after an additional $200 \mathrm{kRad}$ of proton irradiation of the RTP crystal.

Besides the "infant mortality" of the diode lasers, the second-most critical degradation of the BELA laser system has been observed following gamma and proton irradiation of the Nd:YAG laser rod. In particular, the degradation results differed appreciably depending on whether the BELA laser performance has been measured in the pulsed operation mode or in a quasi-continuous mode. This observation

Table 5. LDA Degradation after Proton Irradiation

\begin{tabular}{lcc}
\hline Fluence $\left[10^{11} \mathrm{~cm}^{-2}\right]$ & Energy $[\mathrm{keV}]$ & Degradation [\%] \\
\hline 3 & $70 / 130 / 190^{a}$ & $-0.3 \pm 0.6$ \\
1 & 6000 & $-0.16 \pm 0.05$ \\
3 & 6000 & $-1.5 \pm 0.6$ \\
\hline
\end{tabular}

${ }^{a} 1 \times 10^{11} \mathrm{~cm}^{-2}$ at each energy. 

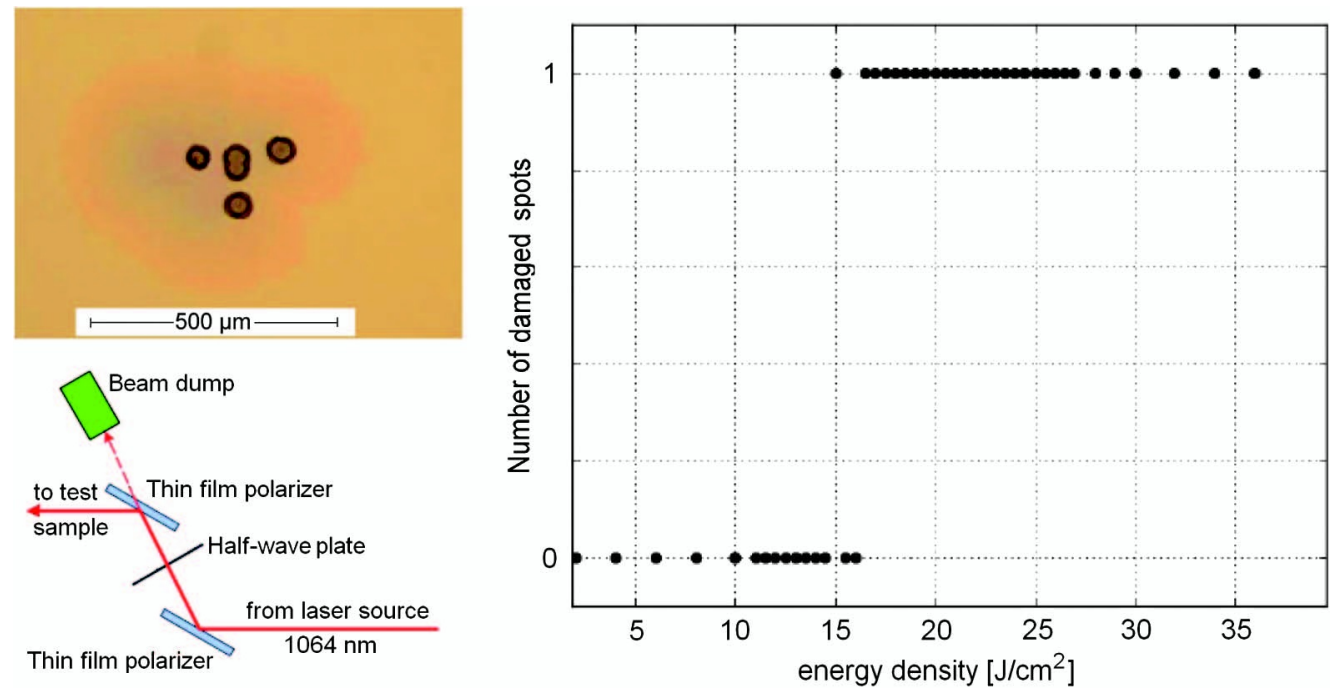

Fig. 8. Upper left: microscope picture of coating damage after exposure of the polarizing beam splitter as test sample (lower left) to subsequently increasing energy densities at $1064 \mathrm{~nm}$ wavelength. Right: at each energy density level, this particular sample has been exposed to 10,000 shots at each spot [57]. The plot simply indicates whether a spot has been damaged within these 10,000 shots or not. This results in an LIDT of approximately $13 \mathrm{~J} \mathrm{~cm}^{-2}$.

has already been made before [58]; however, the underlying physics has not yet been unambiguously resolved. In the representative pulsed operation mode, a degradation of $8.3 \%$ has been measured after $200 \mathrm{kRad}$ of gamma irradiation and a total degradation $11.3 \%$ has been measured following an additional $200 \mathrm{kRad}$ of proton irradiation (Fig. 9, right). Even this degradation could be compensated by adaptation of the pump time from typically $125 \mu \mathrm{s}$ up to $200 \mu$ s. However, the BELA Nd:YAG laser rod only experiences up to $20 \mathrm{kRad}$ of irradiation during the full mission duration of BepiColombo at its well-shielded location inside the LHB.

A critical technological task that had to be solved was the development of a polarizing beam splitter appropriate for the BELA laser resonator. This beam splitter must withstand energy densities of up to $3 \mathrm{~J} \mathrm{~cm}^{-2}$. The achievement of an LIDT of $15 \mathrm{~J} \mathrm{~cm}^{-2}$ has been required internally to give sufficiently large margin for safe operation. This high LIDT is rather hard to achieve because the high extinction ratio of the polarizing beam splitter requires a large number of dielectric coating layers, which again increases the probability of the development of some defect in the coatings. During LIDT testing, particular attention was focused on any outgassing from the adhesives used which could play a critical role under sealed box conditions inside the LHB. As additional reliability measure, synthetic air was chosen for the LHB gas filling already mentioned above. In course of the development of this polarizing beam splitter, three types of dielectric layer materials have been used in combination with $\mathrm{SiO}_{2}$ layers: (1) ion-beam sputtered (IBS) $\mathrm{ZrO}_{2}$, (2) IBS $\mathrm{HfO}_{2}$, and (3) electron-beam evaporated $\mathrm{Ta}_{2} \mathrm{O}_{5}$. With our suppliers the highest LIDT thresholds have been achieved with solution (3) as presented in Fig. 8. The internal requirement of $15 \mathrm{~J} \mathrm{~cm}^{-2}$ has not fully been met, but the achieved safety factor 4 in LIDT turned out to be sufficient because the BELA laser life model has reached the required 300 MShots without any indication of degradation of the polarizing beam splitter. Solution (3) is difficult for assembly and integration because the $\mathrm{Ta}_{2} \mathrm{O}_{5}$ coating needs to remain in dry environment. This is given as soon as the polarizing beam splitter is inside the encapsulated volume of the LHB filled with dry synthetic air. Note that our suppliers have participated in a competition to achieve the highest
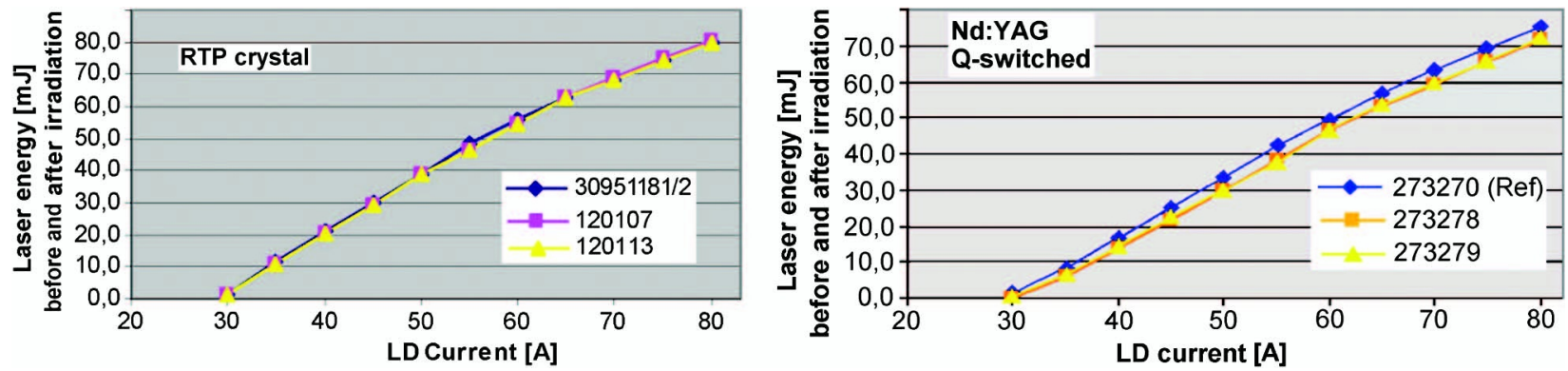

Fig. 9. Left: laser pulse energy before irradiation of the RTP crystal (reference 30951181/2), after 200 kRad of proton irradiation (120107), and after additional $200 \mathrm{kRad}$ of gamma irradiation (120113). Right: laser pulse energy before irradiation of the Nd:YAG laser rod (reference 273270), after $200 \mathrm{kRad}$ of gamma irradiation (273278), and after additional $200 \mathrm{kRad}$ of proton irradiation (273279). 
LIDT of a Brewster angle polarizing beam splitter operating at a wavelength of $1064 \mathrm{~nm}$ [59]. However, the minimum transmission of $98 \%$ required for the BELA application is higher, i.e., more demanding than that required in the mentioned competition $(95 \%)$, as is the reflection value of $99.5 \%$ (99\% in the competition, respectively).

\section{Structural and Thermal Testing}

Having qualified the optoelectronic components for space, the structural and thermal stability and pointing accuracy of the BELA instrument as a whole and of the laser system in particular has received particular attention. The stand-alone LHB had been developed as a hard-mounted device on a very rigid nickel-iron alloy (INVAR) baseplate, and the structural tests at laser level had to be performed in this configuration. The structural modes of the integrated BELA instrument with receiver and laser system mounted onto the rather soft carbon-fiber-enforced aluminum honeycomb baseplate of BELA are very different from the eigenmodes of the stand-alone LHB. Thus, to ensure safe integration of the LHB into the BELA instrument, appropriate structural tests needed to be devised. The adopted approach has been to run a coupled analysis of the integrated system and to infer acceleration values at about 10 different locations of the LHB, such as the footpoints of the LHB bipods on the baseplate, the base unit of the LHB, or the front lens of the BEX. The coupled analysis is based on the vibration loads required by the ESA Experiment Interface Document (EID-A) to the three isostatic mounting interface points of the BELA baseplate to the spacecraft optical bench shown in Fig. 10 (left) as blue line. In fact, the original calculations had been performed with higher input levels indicated by the thin blue line. Only recently, the vibration levels had been relaxed by the ESA down to the levels indicated by the thick blue line in Fig. 10 (left).

The LHB had been designed assuming input levels twice as high as those indicated in Fig. 10 (right), namely at random loads up to $0.5 \mathrm{~g}^{2} \mathrm{~Hz}^{-1}$ at the fundamental LHB resonance at about $275 \mathrm{~Hz}$. The structural thermal model (STM) in fact withstood these loads, which led to rms accelerations of up to 83.3 and $70.7 \mathrm{~g}$ at the BEX front lens and LHB base unit, respectively. The BELA EQM of the LHB has withstood acceleration values up to 64.2 and $57.0 \mathrm{~g}$ at the BEX front lens and LHB base unit, respectively, with all performance parameters unchanged except for bore sight deviating by about $100 \mu \mathrm{rad}$ after random vibration. This change in bore sight has been attributed to slipping and settling at the bipod mounting interface to the BELA baseplate. At reduced rms levels of $9.1 \mathrm{~g}$ at the LHB bipod footpoints at the interface to the BELA baseplate, the BEX lens experienced $40.3 \mathrm{~g}$ (Fig. 10, right) and the bore sight remained within required limits. The originally specified rms acceleration at the BEX front lens was $43.6 \mathrm{~g}$ (Fig. 10, left); however, the recent relaxation of the EID-A loads made the LHB structurally compliant without any notching in the random spectra, i.e., the rms acceleration value of $40.3 \mathrm{~g}$ at the BEX front lens is sufficient.

Interface shear and tension forces at the LHB bipod mounting interfaces on the BELA baseplate have been tested up to $1578 \mathrm{~N}(3 \sigma)$ and $884 \mathrm{~N}$, respectively, while the original requirement of 1307 and $841 \mathrm{~N}$, respectively, from the coupled structural analysis of the BELA instrument could be somewhat relaxed in the mean time due to the reduced loads in the EID-A. The EQM bipod stress has been driven up to $675 \mathrm{MPa}$ (even $964 \mathrm{MPa}$ for the STM), while at most $386 \mathrm{MPa}\left(1 \mathrm{~Pa}=1 \mathrm{Nm}^{-2}\right)$ are required from the coupled analysis. The LHB base unit stress has been tested for 144 and $205 \mathrm{MPa}$ for the EQM and STM, respectively, while the requirement can now be relaxed below the original value of $123 \mathrm{MPa}$.

Bore-sight stability remained the most critical parameter during qualification testing. The boresight changes caused by random vibration testing, shock testing, reorientation of the LHB in Earth's gravity field, and during thermal cycling (Fig. 11) resulted in the bore-sight budget as summarized
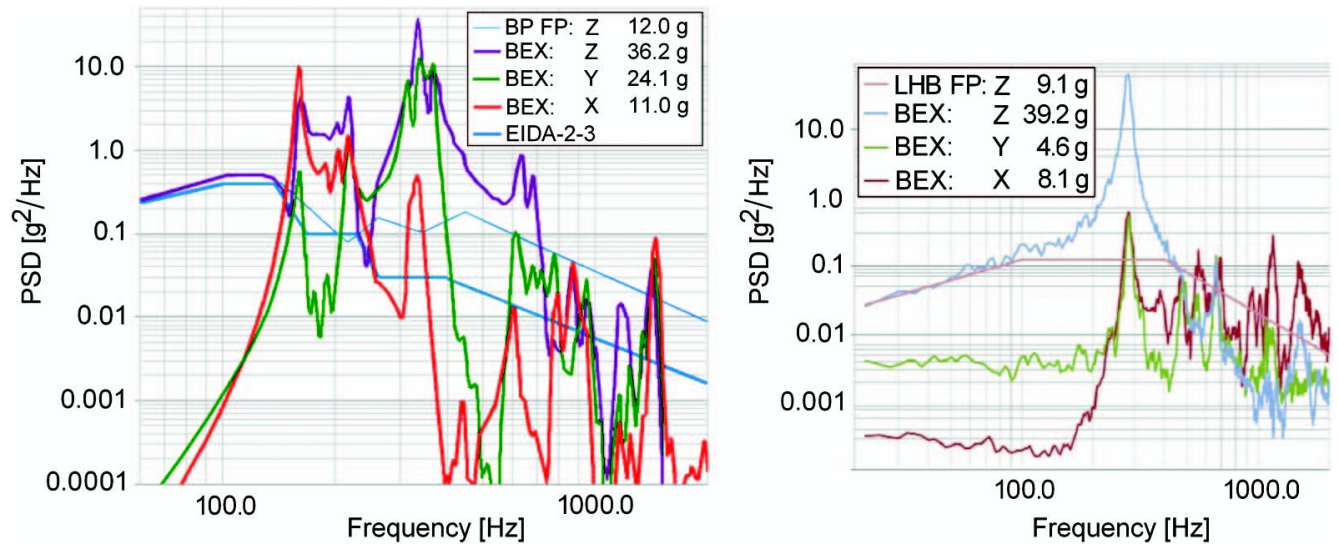

Fig. 10. Left: acceleration values at the BELA BEX from coupled structural analysis for the transmitter and receiver integrated onto the baseplate based on the input to the baseplate footpoints (BP FP) indicated by the thin blue line. Right: measurement results from a random vibration test with the input at the hard-mounted footpoints of the LHB bipods (LHB FP) indicated by the light-brown line. For axis denotations please refer to Fig. 1 . 

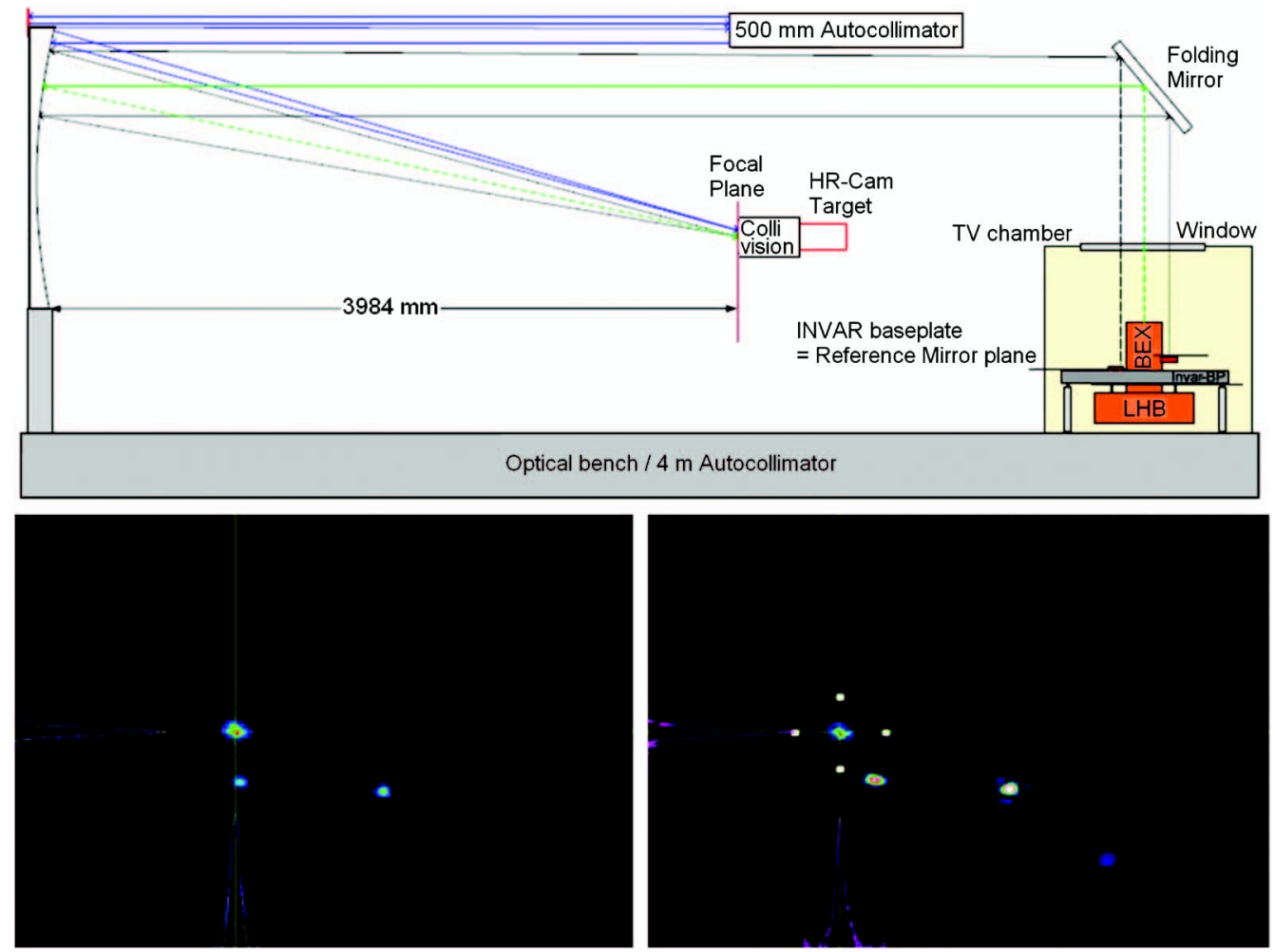

Fig. 11. Top: schematic of measurement setup to record the bore-sight stability of the laser beam. The measurement accuracy of this setup is of order $5 \mu \mathrm{rad}$, where the repeatability of the measurement contributes $5 \mu \mathrm{rad}$, the focal plane adjustment accuracy is $1.7 \mu \mathrm{rad}$, and one of the $1280 \times 1024$ CCD camera pixels corresponds to $1.12 \mu \mathrm{rad}$. Bottom: spots on the CCD camera before (left) and after (right) random vibration from the three reference mirrors mounted to the hard INVAR baseplate (the third reference hardly visible before vibration) of the stand-alone LHB and from the laser beam.

in Table 6. The test results confirm that the hardmounted BELA laser system marginally fulfills the requirement of $50 \mu \mathrm{rad}$ total deviation in bore sight due to all qualification loads. However, the largest fractional change in bore sight is not attributed to the actual laser hardware but to the INVAR baseplate deformation, an effect that shall be reduced during testing of the flight hardware. The shift of the bipods contributes most to the bore-sight error budget of the laser hardware itself. In fact, the mounting mechanism of the LHB bipods to the BELA instrument baseplate is assumed to be most critical for the coalignment of the LHB to the BELA receiver.

Table 6. LHB Bore-Sight Changes $\Delta_{\mathrm{bs}}$ During Qualification Testing

\begin{tabular}{lc}
\hline Load Contribution & $\Delta_{\mathrm{bs}}[\mu \mathrm{rad}]$ \\
\hline Random vibration total $^{a}$ & 25 \\
Shift of bipods & 23 \\
Shift of reference mirrors & 8 \\
Rotation in Earth's gravity field & 11 \\
Shock (1000 g) & 10 \\
Thermal cycling total $^{b}$ & 44 \\
Baseplate deformation & 42 \\
Shift of reference mirrors & 8 \\
Shift optical/mechanical parts & 10 \\
Total qualification loads & 54 \\
\hline
\end{tabular}

${ }^{a}$ As in Fig. 10 (right).

${ }^{b} \mathrm{In}$ the range $\left(-5^{\circ} \mathrm{C} \ldots+55^{\circ} \mathrm{C}\right)$.
The change of beam divergence during qualification testing has been dominated by increases of up to $8 \mu \mathrm{rad}$ due to vibration loads and $4 \mu \mathrm{rad}$ due to thermal loads, where it should be recalled that the measurement accuracy is of order $5 \mu \mathrm{rad}$.

Last but not least, it needs to be mentioned that the leakage rate of the C-ring sealed LHB has been determined experimentally after vibration of the LHB during thermal cycling in a vacuum chamber. The synthetic air filled into the LHB has an admixture of $1 \% \mathrm{He}$. With the help of a helium leak detector (Alcatel ASM 180), the leakage rate has been determined to vary between $2 \times 10^{-9}$ and $10^{-8}$ mbar $1 \mathrm{~s}^{-1}$ in the temperature range between $-5^{\circ} \mathrm{C}$ and $+55^{\circ} \mathrm{C}$. A He leakage rate of $10^{-6}$ mbar $1 \mathrm{~s}^{-1}$ would correspond to a pressure drop from 1 bar to 900 mbar over the projected mission time. Therefore, we consider the risk as very low that the pressure inside the LHB drops below the critical value governed by the so-called Paschen curve, in our case a few millibars, at which corona discharge at the active $q$-switch and its driver electronics could occur.

\section{Electrical Testing}

The BELA laser EQM has undergone standard tests for electromagnetic compatibility (EMC) to verify compliance with the BepiColombo EID-A requirements by the ESA and in addition to the $\mathrm{AC}$ and DC magnetic cleanliness tests. The latter tests 
were important to show compatibility with the magnetometer MERMAG [28] onboard MPO and have been carried out at the facilities of the corresponding PI institute of the Technical University of Braunschweig.

It has been particularly challenging to reduce the electromagnetic radiation by the $100 \mathrm{~A}$ diode laser current pulses, which have a length of approximately $200 \mu \mathrm{s}$ leading to harmonics of $5 \mathrm{kHz}$ signals in the frequency domain. Double-shielded cables with an appropriate grounding scheme have been applied, and the geometry of the 100 A current path has been optimized in order to reduce the $\mathrm{AC}$ magnetic moment of the circuit. In fact, the current load of up to $1 \mathrm{~A}$ of the capacitor reservoir (Fig. 2 , right) that in turn drives the diode laser current also required a dedicated design in order to reduce EMC emissions. A charge-time regulator has been implemented to avoid switching spikes in this load current. This means that the load current is continuously regulated to an optimum value to load the $300 \mathrm{~V}$ capacitor reservoir just as required in the time interval between two laser pulses. This way, the laser can operate at any PRF between 1 and $10 \mathrm{~Hz}$ without distortions due to current spikes.

Reliable operation of the LDAs has been ensured through partial discharge measurements according to IEC 60270. The LDAs are driven in series leading to voltages of up to $65 \mathrm{~V}$ between the electrodes of the miniaturized diode laser stacks and their housing. A safety factor 2 has been verified by these partial discharge measurements.

The Pockels' cell driver circuit with its cascaded transistors IRHNJ67C30 switching $3 \mathrm{kV}$ as described in Section 2.C.1 has also undergone partial discharge testing according to IEC 60270. After conformal coating with Parylene $\mathrm{C}$, which additionally increases the high-voltage reliability but also increases stray capacitance in the circuit, a rise time of $30 \mathrm{~ns}$ has been realized, which turned out to be acceptable to achieve the required laser pulse energy of $50 \mathrm{~mJ}$.

\section{Conclusion}

The major challenges of high-power lasers for space applications are laser-induced damage on miniaturized intracavity optical components, thermomechanical and structural robustness, space qualification of high-power diode lasers, and limited availability of space-qualified electronics components at high level of miniaturization, while simultaneously meeting the mass and power resource availability. These problem areas have to not only be solved for the BELA laser system, but there is quite some margin in performance to make this laser design or a modification of it applicable for further space missions.

The BELA laser system has been developed in the frame of a research and development contract granted by the Max Planck Institute for Solar System Research (MPS) to Cassidian Optronics GmbH (former Carl Zeiss Optronics GmbH), Oberkochen, Germany. Financial support was provided under grant 50QW0501 on behalf of the DLR Space Administration by the Federal Ministry of Economics and Technology based on legislation by the German Parliament. The European Space Agency ESA has funded the diode laser qualification programme with Cassidian Optronics as prime contractor and has given technical advice and support in particular through Bodo Gramkow, Eamonn Murphy, and Jorge Piris. The BELA laser system has been designed and developed in fulfillment of the requirements of the BELA CoPI institutions, the Physikalisches Institut of the University of Bern (UBE), Switzerland, with CoPI Nicolas Thomas, and the DLR Institute for Planetary Research in Berlin (DLR-PF), Germany, with CoPI Tilman Spohn, under close collaboration of the BELA System Engineering team formed by Daniele Piazza, Thomas Behnke, Simone DelTogno, Kay Lingenauber, and the first author. Many thanks go to all involved collaborators in the BELA team, at Cassidian Optronics, at Semiconductor Devices, and at numerous subcontractor companies.

\section{References}

1. M. L. Stitch, "Laser rangefinding," in Laser Handbook, F. T. Arecchi and E. O. Schulz-Dubois, eds. (North-Holland, 1972), pp. $1745-1804$.

2. T. G. Bergman, Design for Laser Rangefinders (U.S. Naval Ordnance Test Station, 1964).

3. P. A. Forrester and K. F. Hulme, "Laser rangefinder," Opt. Quantum Electron. 13, 259-293 (1981).

4. M. C. Amann, T. Bosch, M. Lescure, R. Myllyla, and M. Rioux, "Laser ranging: a critical review of usual techniques for distance measurement," Opt. Eng. 40, 10-19 (2001).

5. W. L. Sjogren and W. R. Wollenhaupt, "Lunar shape via the Apollo laser altimeter," Science 179, 275-278 (1973).

6. D. E. Smith, M. T. Zuber, G. A. Neumann, and F. G. Lemoine, "Topography of the Moon from the Clementine LIDAR," J. Geophys. Res. 102, 1591-1611 (1997).

7. H. Araki, M. Ooe, T. Tsubokawa, N. Kawano, H. Hanada, and $\mathrm{K}$. Heki, "Lunar laser altimetry in the SELENE project," Adv. Space Res. 23, 1813-1816 (1999).

8. M. T. Zuber, D. E. Smith, R. Zellar, G. A. Neumann, X. Sun, J. Connelly, A. Matuszeski, J. F. McGarry, M. Ott, L. Ramos-Izquierdo, D. D. Rowlands, T. Zagwodzki, and M. H. Torrence, "The Lunar Reconnaissance Orbiter laser ranging investigation," Space Sci. Rev. 150, 63-80 (2010).

9. J. A. Kamalakar, K. V. S. Bhaskar, A. S. Laxmi Prasad, R. Ranjith, K. A. Lohar, R. Venketeswaran, and T. K. Alex, "Lunar ranging instrument for Chandrayaan-1," J. Earth Syst. Sci. 114, 725-731 (2005).

10. C. Li, X. Ren, J. Liu, X. Zou, L. Mu, J. Wang, R. Shu, Y. Zou, H. Zhang, C. Lü, J. Liu, W. Zuo, Y. Su, W. Wen, W. Bian, M. Wang, C. Xu, D. Kong, X. Wang, F. Wang, L. Geng, Z. Zhang, L. Zheng, $\mathrm{X}$. Zhu, J. Li, and Z. Ouyang, "Laser altimetry data of Chang-E-1 and the global lunar DEM model," Sci. China: Earth Sci. 53, 1582-1593 (2010).

11. J. L. Bufton, Environmental Assessment: Shuttle Laser Altimeter Experiment (The Center, 1995).

12. B. E. Schutz, "Laser altimetry and lidar from ICESat/GLAS," IEEE Geosci. Remote Sens. 3, 1016-1019 (2001).

13. L. Ramos-Izquierdo, J. L. Bufton, and P. Hayes, "Optical system design and integration of the Mars Observer Laser Altimeter," Appl. Opt. 33, 307-322 (1994).

14. M. T. Zuber, D. E. Smith, A. F. Cheng, and T. D. Cole, "The NEAR laser ranging investigation,” J. Geophys. Res. 102, 23761-23774 (1997).

15. M. T.Zuber, D. E. Smith, S. C. Solomon, R. J. Phillips, S. J. Peale, J. W. Head, S. A. Hauck, R. L. McNutt, J. Oberst, G. A. Neumann, F. G. Lemoine, X. Sun, O. Barnouin-Jha, and 
J. K. Harmon, "Laser altimeter observations from MESSENGER's first Mercury flyby," Science 321, 77-79 (2008).

16. L. Ramos-Izquierdo, S. Scott III, S. Schmidt, J. Britt, W. Mamakos, R. Trunzo, J. Cavanaugh, and R. Miller, "Optical system design and integration of the Mercury Laser Altimeter," Appl. Opt. 44, 1748-1760 (2005).

17. D. J. Krebs, A.-M. Novo-Gradac, S. X. Li, S. J. Lindauer, R. S. Afzal, and A. W. Yu, "Compact, passively $q$-switched Nd:YAG laser for the MESSENGER mission to Mercury," Appl. Opt. 44, 1715-1718 (2005).

18. D. L. Domingue and C. T. Russell, eds., The MESSENGER Mission to Mercury (Springer, 2008).

19. R. E. Gold, S. C. Solomon, R. L. McNutt, Jr., A. G. Santo, J. B. Abshire, M. H. Acũna, R. S. Afzal, B. J. Anderson, G. B. Andrews, P. D. Bedini, J. Cain, A. F. Cheng, L. G. Evans, W. C. Feldman, R. B. Follas, G. Gloeckler, J. O. Goldsten, S. E. Hawkins, N. R. Izenberg, S. E. Jaskulek, E. A. Ketchum, M. R. Lankton, D. A. Lohr, B. H. Mauk, W. E. McClintock, S. L. Murchie, C. E. Schlemm, D. E. Smith, R. D. Starr, and T. H. Zurbuchen, "The MESSENGER mission to Mercury: scientific payload," Planet. Space Sci. 49, 1467-1479 (2001).

20. A. Balogh, R. Grard, S. C. Solomon, R. Schulz, Y. Langevin, Y. Kasaba, and M. Fujimoto, "Missions to Mercury," Space Sci. Rev. 132, 611-645 (2007).

21. S. C. Solomon, R. L. McNutt, Jr., R. E. Gold, M. H. Acũna, D. N. Baker, W. V. Boynton, C. R. Chapman, A. F. Cheng, G. Gloeckler, J. W. Head, S. M. Krimigis, W. E. McClintock, S. L. Murchie, S. J. Peale, R. J. Phillips, M. S. Robinson, J. A. Slavin, D. E. Smith, R. G. Strom, J. I. Trombka, and M. T. Zuber, "The MESSENGER mission to Mercury: scientific objectives and implementation," Planet. Space Sci. 49, 14451465 (2001)

22. N. Thomas, T. Spohn, J.-P. Barriot, W. Benz, G. Beutler, U. Christensen, V. Dehant, C. Fallnich, D. Giardini, O. Groussin, K. Gunderson, E. Hauber, M. Hilchenbach, L. Iess, P. Lamy, L.-M. Lara, P. Lognonné, J. J. Lopez-Moreno, H. Michaelis, J. Oberst, D. Resendes, J.-L. Reynaud, R. Rodrigo, S. Sasaki, K. Seiferlin, M. Wieczorek, and J. Whitby, "The BepiColombo Laser Altimeter (BELA): concept and baseline design," Planet. Space Sci. 55, 1398-1413 (2007).

23. K. Seiferlin, S. Chakraborty, K. Gunderson, J. Fischer, B. Lüthi, D. Piazza, M. Rieder, M. Sigrist, N. Thomas, and T. Weigel, "Design and manufacture of a lightweight reflective baffle for the BepiColombo Laser Altimeter," Opt. Eng. 46, 043003 (2007).

24. T. Beck, A. Bieler, and N. Thomas, "Numerical thermal mathematical model correlation to thermal balance test using adaptive particle swarm optimization (APSO)," Appl. Therm. Eng. 38, 168-174 (2012).

25. S. Chakraborty, M. Affolter, K. Gunderson, J. Neubert, N. Thomas, T. Beck, M. Gerber, S. Graf, D. Piazza, A. Pommerol, G. Roethlisberger, and K. Seiferlin, "High accuracy alignment facility for the receiver and transmitter of the BepiColombo Laser Altimeter," Appl. Opt. 51, 4907-4915 (2012).

26. A. Milani, A. Rossi, D. Vokrouhlicky, D. Villani, and C. Bonanno, "Gravity field and rotation state of Mercury from the BepiColombo radio science experiments," Planet. Space Sci. 49, 1579-1596 (2001).

27. D. M. Lucchesi and V. Iafolla, "The non-gravitational perturbations impact on the BepiColombo radio science experiment and the key role of the ISA accelerometer: direct solar radiation and albedo effects," Celestial Mech. Dyn. Astron. 96, 99-127 (2006).

28. K.-H. Glassmeier, H.-U. Auster, D. Heyner, K. Okrafka, C. Carr, G. Berghofer, B.-J. Anderson, A. Balogh, W. Baumjohann, P. Cargill, U. Christensen, M. Delva, M. Dougherty, K.-H. Fornacon, T.-S. Horbury, E.-A. Lucek, W. Magnes, M. Mandea, A. Matsuoka, M. Matsushima, U. Motschmann, R. Nakamura, Y. Narita, H. O'Brien, I. Richter, K. Schwingenschuh, H. Shibuya, J.-A. Slavin, C. Sotin, B. Stoll, H. Tsunakawa, S. Vennerstrom, J. Vogt, and T. Zhang, "The fluxgate magnetometer of the BepiColombo Mercury Planetary Orbiter," Planet. Space Sci. 58, 287-299 (2010).

29. M. Sgavetti, L. Pompilio, C. Carli, M. C. de Sanctis, F. Capaccioni, G. Cremonese, and E. Flamini, "VIHI: the Visible and Infrared Hyperspectral Imager channel of the SIMBIOSYS instrument for the BepiColombo mission to Mercury," Planet. Space Sci. 55, 1596-1613 (2007).

30. U. R. Christensen, "A deep dynamo generating Mercury's magnetic field," Nature 444, 1056-1058 (2006).

31. C. Koch, U. R. Christensen, and R. Kallenbach, "Simultaneous determination of global topography, tidal Love number and libration amplitude of Mercury by laser altimetry," Planet. Space Sci. 56, 1226-1237 (2008).

32. T. Van Hoolst and C. Jacobs, "Mercury's tides and interior structure," J. Geophys. Res. 108, 5121 (2003).

33. C. S. Gardner, "Ranging performance of satellite laser altimeters," IEEE Trans. Geosci. Remote Sens. 30, 1061-1072 (1992).

34. W. Riede, P. Allenspacher, and L. Jensen, "Analysis of the air-vacuum effect in dielectric coatings," Proc. SPIE 7132, $71320 \mathrm{~F}$ (2008).

35. K. Weidlich, M. Rech, and R. Kallenbach, "Qualification testing of the laser transmitter part for ESA's BepiColombo Laser Altimeter (BELA)," Proc. SPIE 8159, 815906 (2011).

36. T. D. Cole, "Spaceborne laser altimetry," Crit. Rev. 66, 383-405 (1996).

37. A. F. Cheng, T. D. Cole, M. T. Zuber, D. E. Smith, Y. Guo, and F. Davidson, "In-flight calibration of the near earth asteroid rendezvous laser rangefinder," Icarus 148, 572-586 (2000).

38. K. Marti, "Analysis of avalanche photodiodes and the rangefinder electronics for the Bepicolombo Laser Altimeter (BELA)," Ph.D. Thesis (University of Bern, 2010).

39. T. McCord and J. Adams, "Mercury: surface composition from the reflections spectrum," Science 178, 745-747 (1972).

40. B. Hapke, "Space weathering from Mercury to the asteroid belt," J. Geophys. Res. 106, 10039-10074 (2001).

41. M. T. Zuber, D. E. Smith, R. J. Phillips, S. C. Solomon, G. A. Neumann, S. A. Hauck, S. J. Peale, O. S. Barnouin, J. W. Head, C. L. Johnson, F. G. Lemoine, E. Mazarico, X. Sun, M. H. Torrence, A. M. Freed, C. Klimczak, J.-L. Margot, J. Oberst, M. E. Perry, R. L. McNutt, J. A. Balcerski, N. Michel, M. J. Talpe, and D. Yang, "Topography of the northern hemisphere of Mercury from MESSENGER Laser Altimetry," Science 336, $217-220$ (2012).

42. K. Gunderson, N. Thomas, and M. Rohner, "A laser altimeter model and its application to BELA," IEEE Trans. Geosci. Remote Sens. 44, 3308-3319 (2006).

43. C. S. Gardner, "Target signatures for laser altimeters: an analysis," Appl. Opt. 21, 448-453 (1982).

44. H. Becht, H. Hubach, M. Rech, and M. Weispfennig, "Efficient lasers for remote sensing," Proc. SPIE 7686, 76860D (2010).

45. C. Zimmermann, R. Kallenbach, T. W. Hänsch, and J. Sandberg, "Doubly-resonant second-harmonic generation in $\beta$-barium-borate," Opt. Commun. 71, 229-234 (1989).

46. R. Kallenbach, C. Zimmermann, D. H. McIntyre, T. W. Hänsch, and R. G. Devoe, "A blue dye laser with sub-kilohertz stability," Opt. Commun. 70, 56-60 (1989).

47. C. Zimmermann, R. Kallenbach, and T. W. Hänsch, "Highresolution spectroscopy of the hydrogen $1 \mathrm{~S}-2 \mathrm{~S}$ transition in an atomic beam," Phys. Rev. Lett. 65, 571-574 (1990).

48. W. Riede, H. Schroeder, P. Allenspacher, and P. Mahnke, "Aspects of laser optics qualification for space applications," Proc. SPIE 7504, 75040T (2009).

49. W. Riede, H. Schroeder, G. Bataviciute, D. Wernham, A. Tighe, F. Pettazzi, and J. Alves, "Laser-induced contamination on space optics," Proc. SPIE 8190, 81901E (2011).

50. D. M. Winker, W. H. Hunt, and M. J. McGill, "Initial performance assessment of CALIOP," Geophys. Res. Lett. 34, L19803 (2007).

51. G. Klumel, Y. Karni, S. Cohen, M. Rech, and K. Weidlich, "Temperature and current accelerated lifetime conditions and testing of laser diodes for ESA BepiColombo space mission," Proc. SPIE 7918, 791804 (2011).

52. F. X. Daiminger, F. Dorsch, and S. Heinemann, "Aging properties of $\mathrm{AlGaAs} / \mathrm{GaAs}$ high power diode lasers," Proc. SPIE 3244, 587-595 (1998).

53. B. R. Lawn, Fracture of Brittle Solids (Cambridge University, 1995)

54. J. Piris, "BELA laser diode shock test: post-shock report," LDTF-BELA-TR-002 (ESA ESTEC test report, 2012). 
55. J. F. Ziegler, J. P. Biersack, and U. Littmark, The Stopping and Range of Ions in Solids (Pergamon, 1985).

56. P. Sigmund, Stopping of Heavy Ions: A Theoretical Approach, Springer Tracts in Modern Physics (Springer, 2004), Vol. 204.

57. A. Meissner, "LIDT test protocol," BC-BEL-TR-62525-1-0 (Fraunhofer Institute for Laser Technology, ILT, 2011).
58. T. S. Rose, M. S. Hopkins, and R. A. Fields, "Characterization and control of gamma and proton radiation effects on the performance of Nd:YAG and Nd:YLF lasers," IEEE J. Quantum Electron. 31, 1593-1602 (1995)

59. C. J. Stolz and J. Runkel, "Brewster angle polarizing beamsplitter laser damage competition: P polarization," Proc. SPIE 8530, 85300M (2012). 